In G. Borriello and L.E. Holmquist (Eds.): UbiC omp 2002,

LNC S 2498, pp. 16-32, Springer, 2002.

Presented at U bicomp 2002, Sep 29 - O ct 1, 2002, Göteborg, Sweden.

\title{
ComicDiary: Representing Individual Experiences in a Comics Style
}

\author{
Yasuyuki Sumi†, Ryuuki Sakamotoł, Keiko Nakao†, and Kenji Mase† \\ $\dagger$ ATR Media Information Science Laboratories, \\ Seika-cho, Soraku-gun, Kyoto 619-0288, Japan \\ $\ddagger J a p a n$ Advanced Institute of Science and Technology, \\ 1-1 Asahidai, Tatsunokuchi, Ishikawa 923-1292 Japan \\ sumi@atr.co.jp, http://www2.mis.atr.co.jp/comicdiary/
}

\begin{abstract}
This paper describes a system called ComicDiary that automatically creates a personal diary in a comics style. ComicDiary is built as a sub-system of our ongoing project (C-MAP) to develop a personal guidance system for exhibition touring at museums, trade shows, academic conferences, cities, and so on. The aim of the C-MAP system is to provide users with personalized guidance according to temporal and spatial situations as well as individual interests. ComicDiary is designed as a casual tool for augmenting an individual user's memory as well as for encouraging communities of users to exchange personal memories. This paper presents the preliminary results of deploying the ComicDiary system as a digital assistant service for conference participants.
\end{abstract}

\section{Introduction}

This paper describes the ComicDiary system, which represents individual experiences and interests in comics style. ComicDiary is designed to facilitate knowledge sharing and conversations among community members who share experiences and situations. A diary is fundamentally private: It stimulates personal memories and self-expression and is not supposed to be shown to another person. On the other hand, we intend for diaries made by ComicDiary to be freely shown to other people to increase opportunities to talk about their experiences.

ComicDiary is built as a sub-system of our ongoing project (C-MAP) [1] to develop a personal guidance system for exhibition touring at museums, trade shows, academic conferences, cities, and so on. The aim of the C-MAP system is to provide users with personalized guidance according to temporal and spatial situations as well as individual interests. ComicDiary provides individual users with a tool to record their touring summaries.

The process of generating a comic diary mainly consists of the following two steps. First, ComicDiary determines the story stream of a comic diary from fragmentary data of the user's touring records such as exhibit visiting and interactions with other users (e.g., business card exchange). Second, it renders comic frames for assembling the necessary characters and scene backgrounds. 
In this paper, we describe the implementation and experimental results of making ComicDiary available for use by participants of an academic conference in May 2001. Also, we briefly present another version of ComicDiary as an online service, which was used by participants of another academic conference in August 2001.

\section{Using Comics to Exchange Individual Experiences}

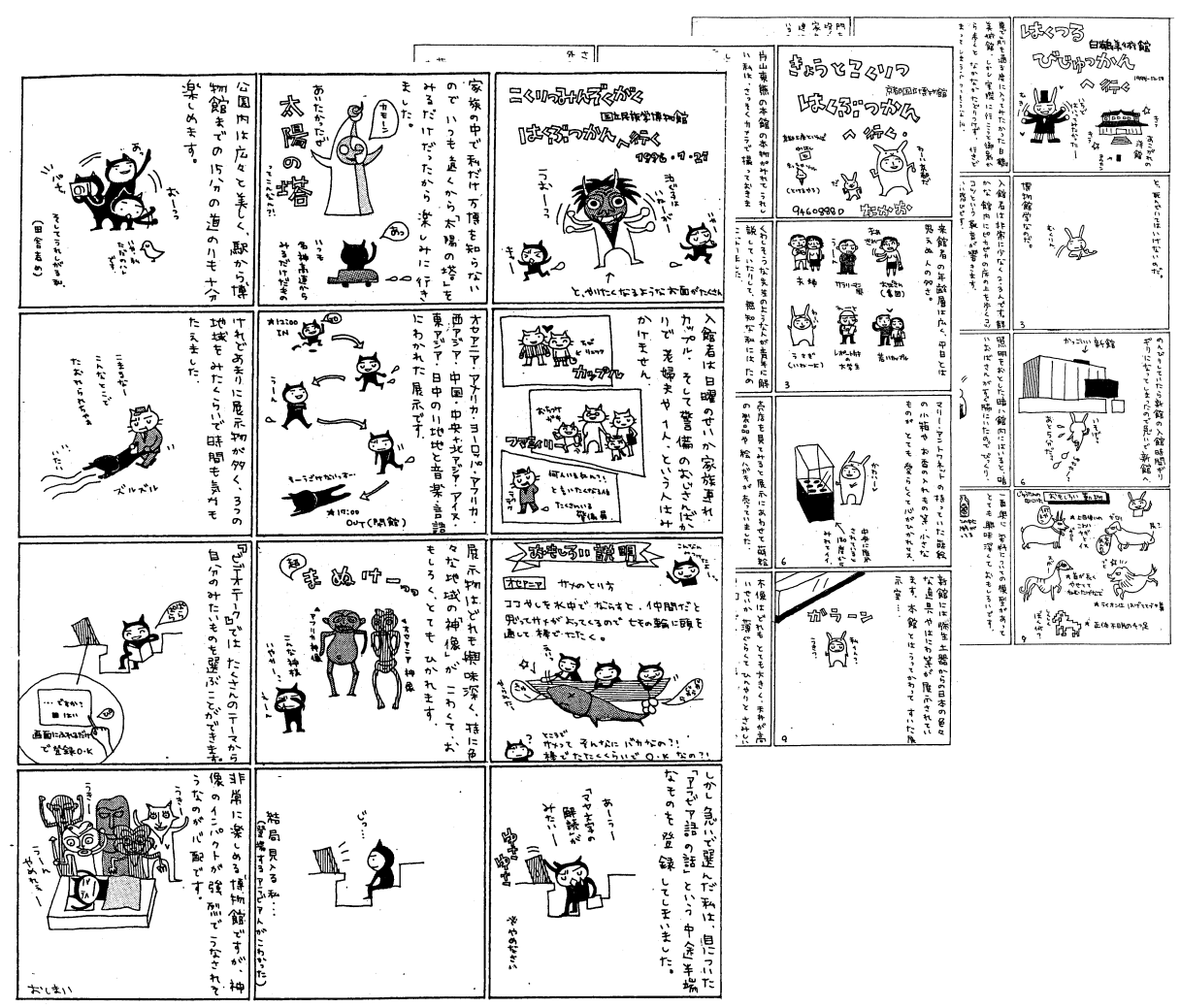

Fig. 1. Examples of hand-drawn diary in comics style.

Fig. 1 shows examples of hand drawn diaries that were drawn and submitted as reports of visiting museums by one of the authors when she took a university class. Other students submitted conventional text-based reports of course, but she was allowed to submit such a comic report because her illustrating skill was admired by the professor.

We can find the following characteristics in the comic report. 
- The story of the comic is structured according to her subjective viewpoint.

- The main character of the comic seems to be herself, but it does not exactly represent her appearance, personality, and actions. The comic character has its own identity and personality as her alter ego.

- The comic exaggerates her personal impressions by projecting them to the alter ego character rather than exactly reproducing actual events.

- The comic highlights impressive events rather than listing every event.

- Her total impression of the museum visit is represented by a rhythm of sequential scenes.

- The comic describes not only the museum's exhibits but also the surroundings and other visitors.

- Jokes and small talk increase the comic's entertainment value.

The comic report is not appropriate for precisely conveying detailed events, but it is excellent for sharing personal impressions and episodes. Actually, the comic reports stimulated the authors' group to exchange individual memories of museum visits and increased their motivation to revisit the museums.

Such experience encouraged us to try a system that provides visit diaries to individual users of our tour guidance system [1]. The technical challenges we addressed were:

- Developing a method to structure a comic story from fragmentary data of individual users (personal profile and visiting records) stored in the tour guidance system and to personalize the comic contents; and

- Prototyping a so-called expert system that emulates the human skill of comic drawing.

\section{Related Works}

Recently, there have been many studies on context-aware applications with ubiquitous and mobile/wearable computing technologies. One goal of such applications is collect and summarize a user's personal history and exploit the results to help the user's recollection [2-5]. In contrast, our aim is to augment not only the user's own memory, but also communications among users. An exception is the Digital Family Portrait project [6], which provides awareness of a family member's daily life and promotes a sense of presence for extended family members. They focused on qualitative visualizations of daily life by using family portraits, while we focus on summarization of sequential events during an exhibition tour by using a comics style. In order to help the user to express his/her memories to others, a comic diary could be effective by prompting them to exchange experiences and interests.

One of our main technological challenges is to allegorize individual fragmentary episodes as a comic story. There have been studies of story generation in the context of artificial intelligence (AI) and cognitive science. Rummelhart [7] tried to build a grammatical schema of stories through analyzing traditional fables. Schank and Abelson [8] regarded AI issues, e.g., problem solving and planning, 
as story generation. Recently, several systems of story generation have been proposed (e.g., $[9,10])$. These works, however, aimed to understand literary works and limited their focus to literal representation. On the other hand, we aim to facilitate human communications through generated stories and employ a comic representation because it is casual and easy to use in showing an overview of personal events.

There have already been some interesting research projects employing comic style as a user interface representation. These include Comic Chat for representing an online chat history as a comic [11], Video Manga for visualizing a video summary in comics style [12], and ChatScape for using captured camera images to represent an online chat history as a comic [13]. Although they used comic representation, these works did not step into translating simple sequences of events into a story or personalizing the generated story; these are the issues that we address here.

\section{System Implementation as a Digital Assistant Service for Conference Participants}

\subsection{Digital Assistant for Conference Participants}

The first prototype of ComicDiary was implemented as our digital assistant system for participants of an academic conference, which was held from May 22 to 25, 2001 in Japan (JSAI 2001). The digital assistant system provided users with a hand-held guidance system (PalmGuide), kiosk services, and meeting facilitator (AgentSalon) for onsite conference participants as well as continuous Web services for pre/post conference in formation [5].

Fig. 2 illustrates the intended usage scenario of ComicDiary. ComicDiary generates a comic diary from user's touring history and records of his/her interactions (virtual business card exchange and accesses to the AgentSalon system) with other users at the conference site, which are recorded and stored on the user's PalmGuide.

\subsection{Accumulation of Personal Information by PalmGuide}

As shown in Fig. 3, PalmGuide is basically a portable browser for showing a conference program. The PalmGuide user can call up his/her personal agent, who recommends upcoming presentations inferred as interesting to the user according to his/her current context (personal preference, current interest, and temporal situation). As feedback for generating knowledge of user interest, users can rate (1: not interesting, 2: average, 3 : interesting) the individual presentations they attend $^{1}$.

\footnotetext{
${ }^{1}$ We could acquire an individual participant's touring history with a location detection system that used an infrared badge/sensor, although we did not use the system in the 2001 version. In the 2000 version, we could collect the users' ratings by using a "marking button" on the back of the badge [5].
} 


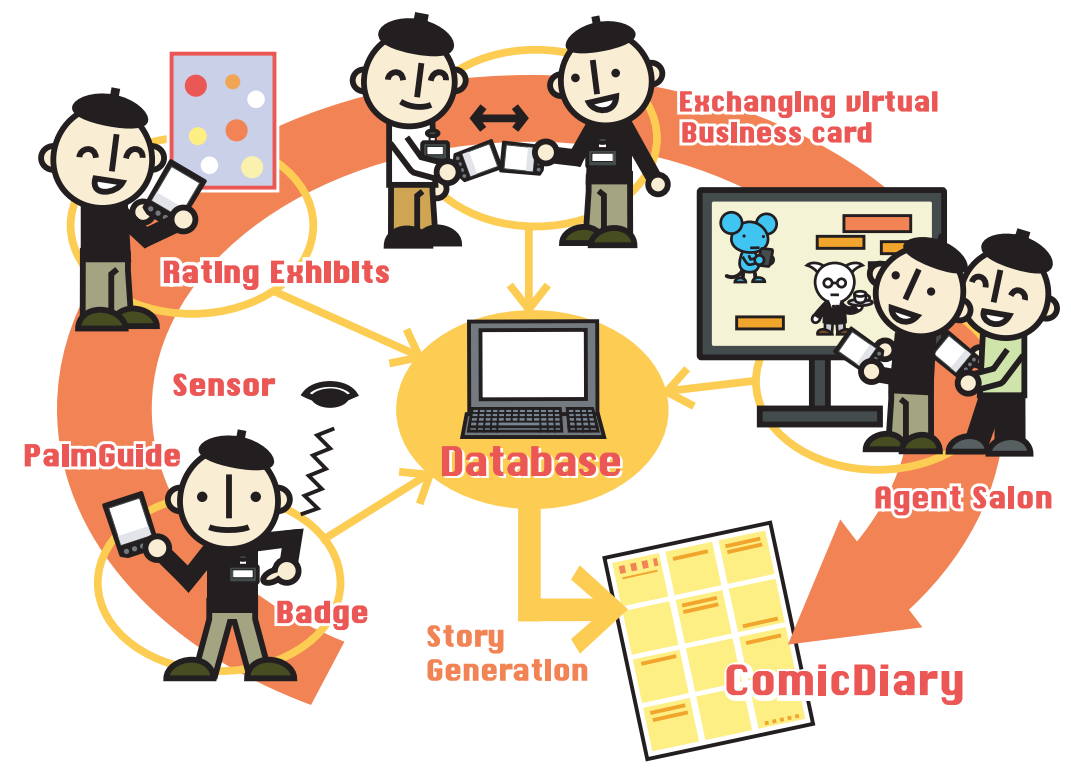

Fig. 2. Scenario of system usage.

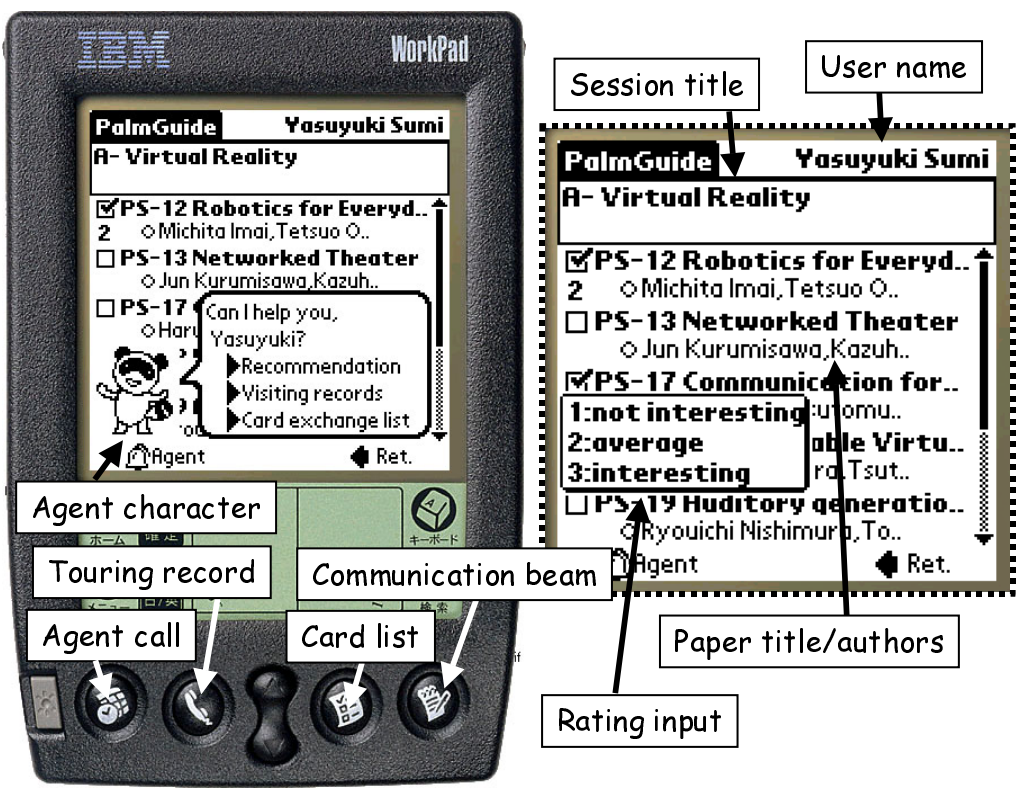

Fig. 3. Accumulation of personal information by PalmGuide. 


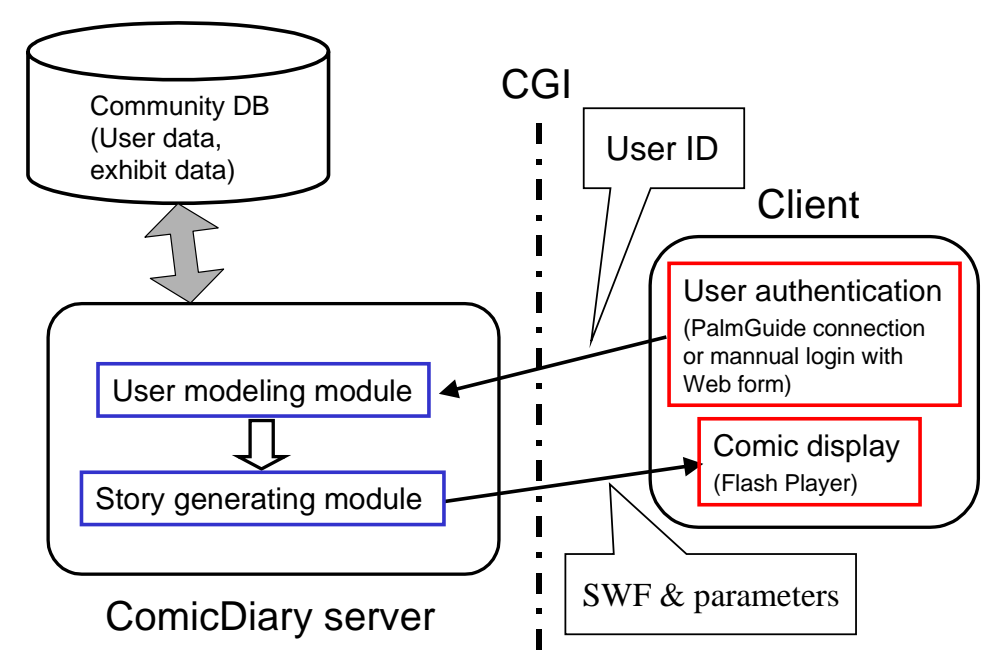

Fig. 4. System architecture.

PalmGuide users can exchange their virtual business cards by infrared connections and use AgentSalon as a facilitator of face-to-face meetings at the conference site by sending their guide agents from PalmGuides to the AgentSalon system. PalmGuide also stores these electronic histories.

The stored data of a personal history is copied to the central community data base (DB) when a user connects his/her PalmGuide to kiosks located at the conference site and when the PalmGuide devices are returned. By centralizing the individual data to the community DB, we can also obtain statistical data such as which presentations are popular among PalmGuide users.

\subsection{System Architecture of ComicDiary}

We prototyped ComicDiary as an online service on the Web so that it could be used as a kiosk service provided at the conference site as well as an online service via the Web that users could access anytime after the conference. We also provided PalmGuide users with printed comic diaries when they returned their PalmGuide devices.

Fig. 4 illustrates the system architecture of ComicDiary. We employed Macromedia Flash running on Web browsers to display the generated comic diaries on client machines. Diary generation was processed at a Web server that communicates with clients via CGI.

The following processes were carried out for diary generation.

1. Clients certify users. When ComicDiary is used by accessing a kiosk at the conference site, a user ID is automatically sent from the PalmGuide to the server via the kiosk. When ComicDiary is used as a Web service, the user logs in to the digital assistant service site with his/her user ID and password. 
2. Requests to generate comic diaries with the user IDs are sent to the server via CGI.

3. The server extracts the user's personal data and statistical data from the community DB and then generates a comic diary story for him/her by determining the user type from the data.

4. A Flash file (SWF) and parameter data for comic rendering are sent to the client.

5. The client renders and displays a comic diary according to the given parameter.

\subsection{Story Generation Engine}

The most important part of ComicDiary is the story generation engine, which is a knowledge-based system. It mainly contains of a user modeling module to collect the necessary data and a story generation module to allegorize a story.

User modeling is done with a personal profile containing attributes of a user and a community profile that is shared by all PalmGuide users. The personal profile includes the following data.

- Age and gender, which are reflected in the personality of the main character of a diary.

- Participant type: Whether they have their own presentations at the conference.

- Touring history (presentations the user attended and their ratings). The data reflects the user's activity level at the conference.

- Interaction records with other PalmGuide users, such as business card exchanges and accesses to AgentSalon.

The user modeling module generates the outline of a diary according to the above data.

The community profile includes the following data.

- Plenary events of the conference, e.g., reception and invited talks.

- Information of the conference venue, e.g., tourist information and current topics.

- Socially shared impressions during the conference, e.g., popularity of presentations.

The above data are used to increase the reality of comic diaries.

Potential streams of a comic story are prepared as a constraint network representing mutual dependencies among scenes as shown in Fig. 5. That is, several alternatives for introductory scenes are followed by presentation scenes (if a user gives a presentation at the conference), touring scenes, interaction scenes (of business card exchanges and/or AgentSalon), and ending scenes. The dependencies (i.e., exclusive relationship and causal relationship) among the scenes were predefined by the comic designer.

An individual scene contains a number of frames. For example, an introductory scene contains three frames, and a presentation scene contains four frames in the first prototype. 


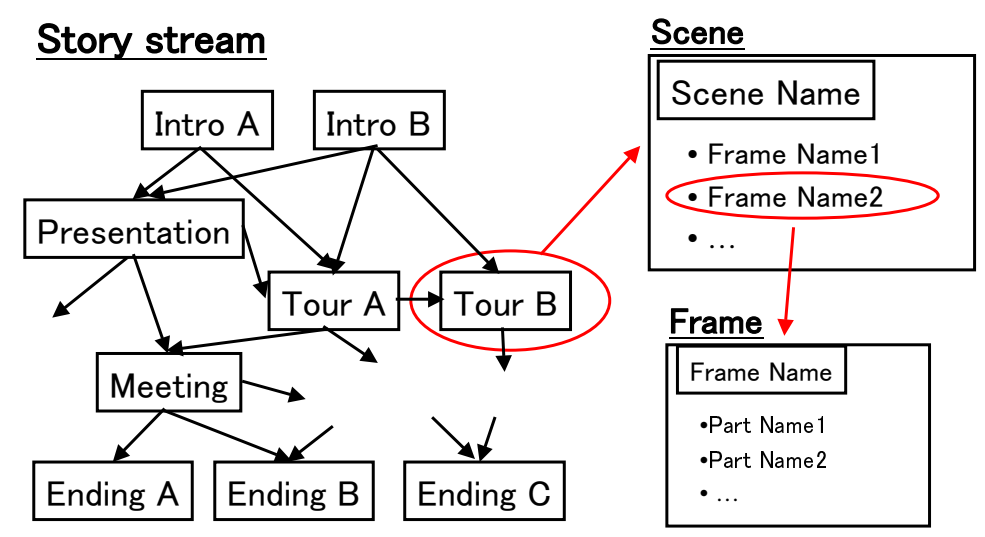

Fig. 5. Story generation of a comic diary.

\subsection{Rendering Comic Frames}

In order to increase the variety of comic frames from limited resources, we employed a method to superimpose several layers as shown in Fig. 6. Accordingly, we could reuse background layers (e.g., scenes of conference rooms) independently of main characters. In the first prototype, we prepared 12 kinds of background layers to show scenes of the conference rooms and sightseeing spots around the conference site.

We prepared 44 illustrations for each character, as shown in Fig. 7, because they greatly influence the entertainment value and variety of comics. The main character was the agent character which user selected for PalmGuide. Since we prepared eight kinds of characters for PalmGuide, we had to prepare 352 appearances of main character layers. That was one of the hardest works for prototyping the ComicDiary system.

Templates of word layers were prepared as images in the same way as the background and main character layers, and strings of dynamically extracted data (e.g., presentation titles and user names) were embedded in the templates during the rendering process.

\section{Comic Examples and User Evaluations}

\subsection{Examples of Generated Comic Diaries}

This section shows examples of generated comic diaries and explains how ComicDiary works. We fixed the length of comics to 12 frames as shown in Fig. 8 because we intended the comics to be printed on A4 size paper. The main character in the comics is the agent character, which the user selects for PalmGuide and we characterize as the user's avatar (closely identified with the user, but, a different person). 


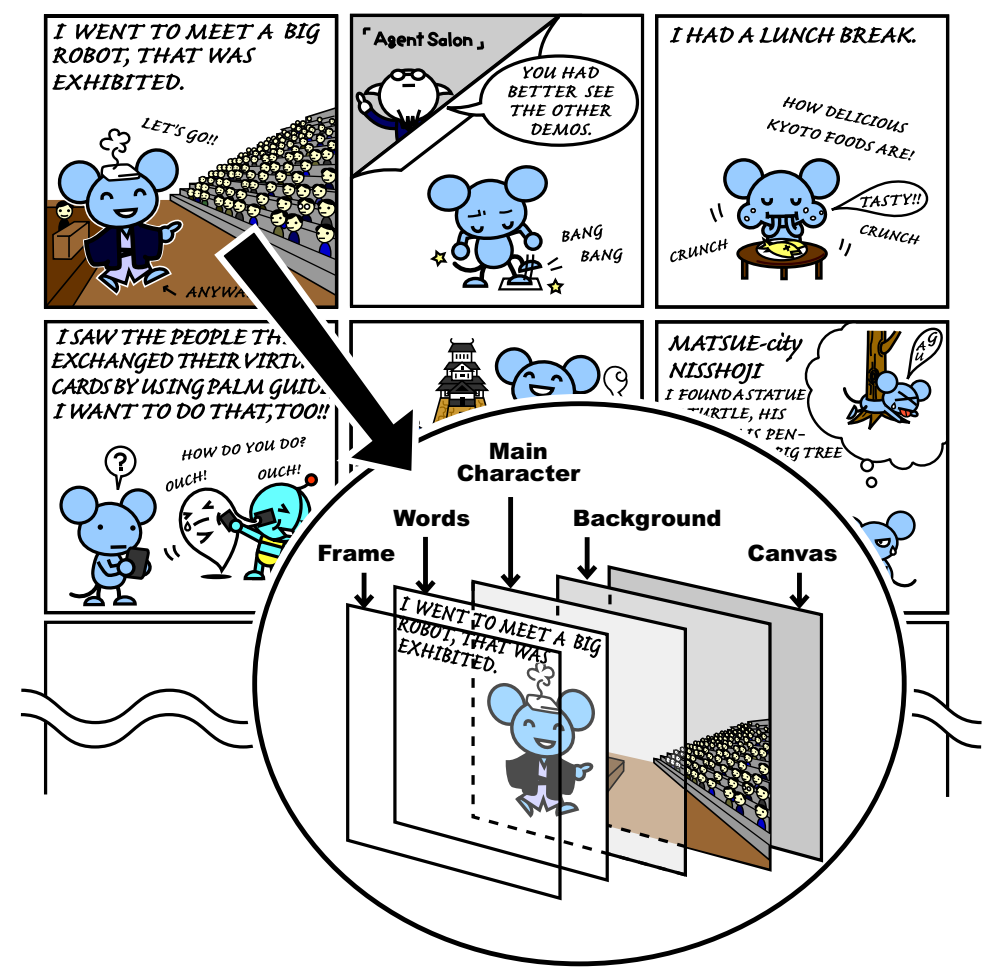

Fig. 6. Frame composition by multiple layers.

We prepared three types of story outlines: for presenters, for active attendees, and for non-active attendees according to the user's personal profile.

Fig. 8 is an example of a comic diary for a user who gives a presentation at the conference. Four frames were used for the user's presentation scene because it was considered the highlight of the user's experiences at the conference. The presentation scene was followed by scenes of meeting with other participants, such as business card exchanges and AgentSalon accesses. Presentation titles and participant names embedded in the comic were extracted from the community DB.

The comic contained not only personal scenes but also environmental scenes to represent the conference atmosphere, e.g., sightseeing spots around the conference site. Also, we provided a scene for giving feedback to the user, e.g., the socially formed impression of the user's presentation according to the statistical results of ratings given on PalmGuides.

For a user who did not give a presentation at the conference, the outline of the comic was drastically changed according to the number of presentations that he/she attended and the average value of the ratings. Fig. 9 is an example comic diary for an active participant and Fig. 10 is one for a non-active participant. 

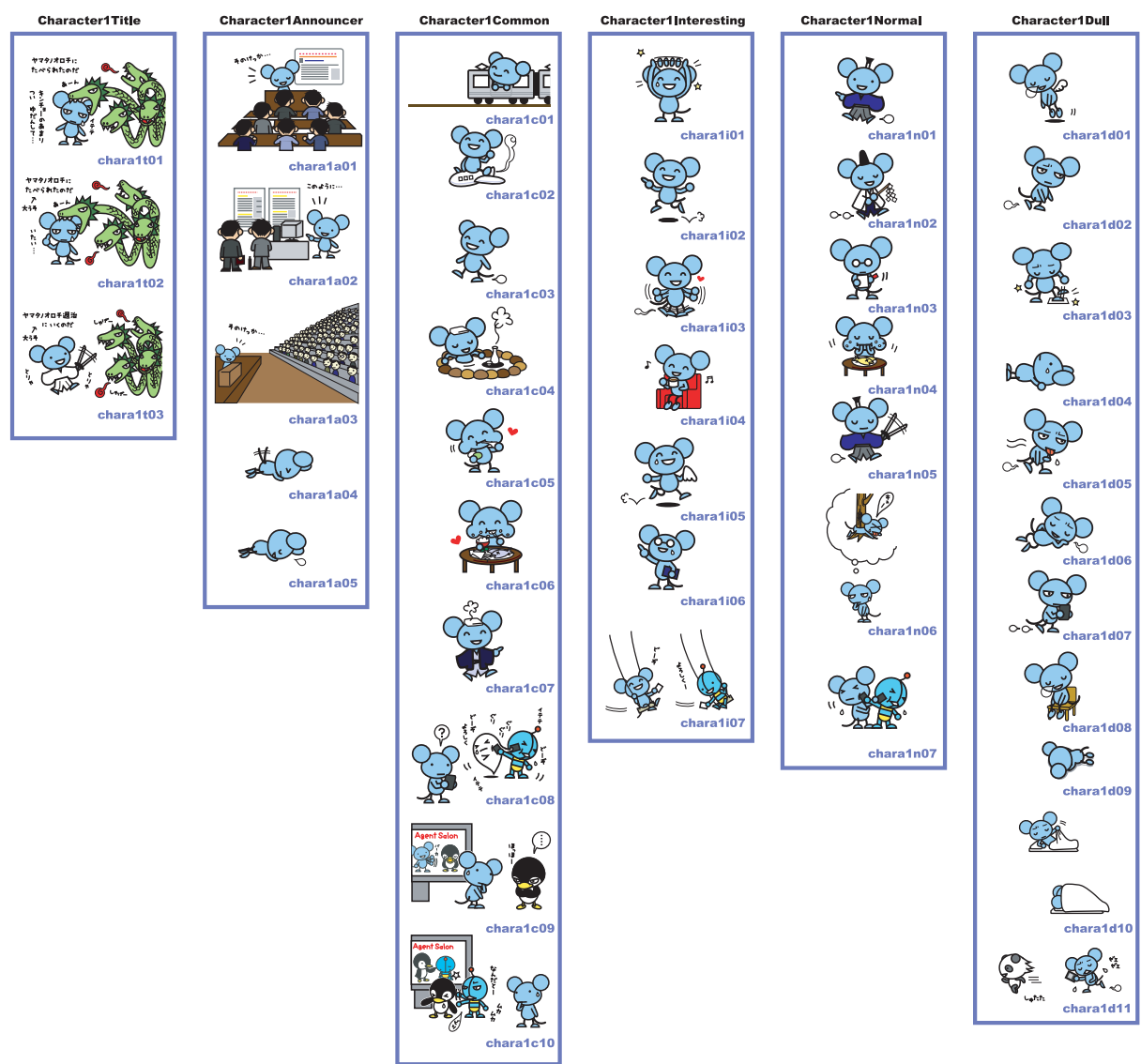

Fig. 7. Comic-part data (of main character layer).

The former comic starts with a cheerful atmosphere and is embedded with many scenes of attending presentations. Embedded presentations were chosen from the presentations that the user evaluated as "interesting." The latter comic, in contrast, was presented with a negative (but amusing) mood.

For both cases, we provided a scene showing popular presentations according to the statistical data accumulated in the community DB during the conference in order to increase the awareness shared by the members of the community.

\subsection{User Evaluations and Discussion}

Fifty-two PalmGuide users participated in the digital assistant project in 2001, and they were ComicDiary users at the same time. We provided printed versions of the generated comic diaries to those who requested them. After five weeks, we asked them to fill out a questionnaire and received replies from 16 . The data 


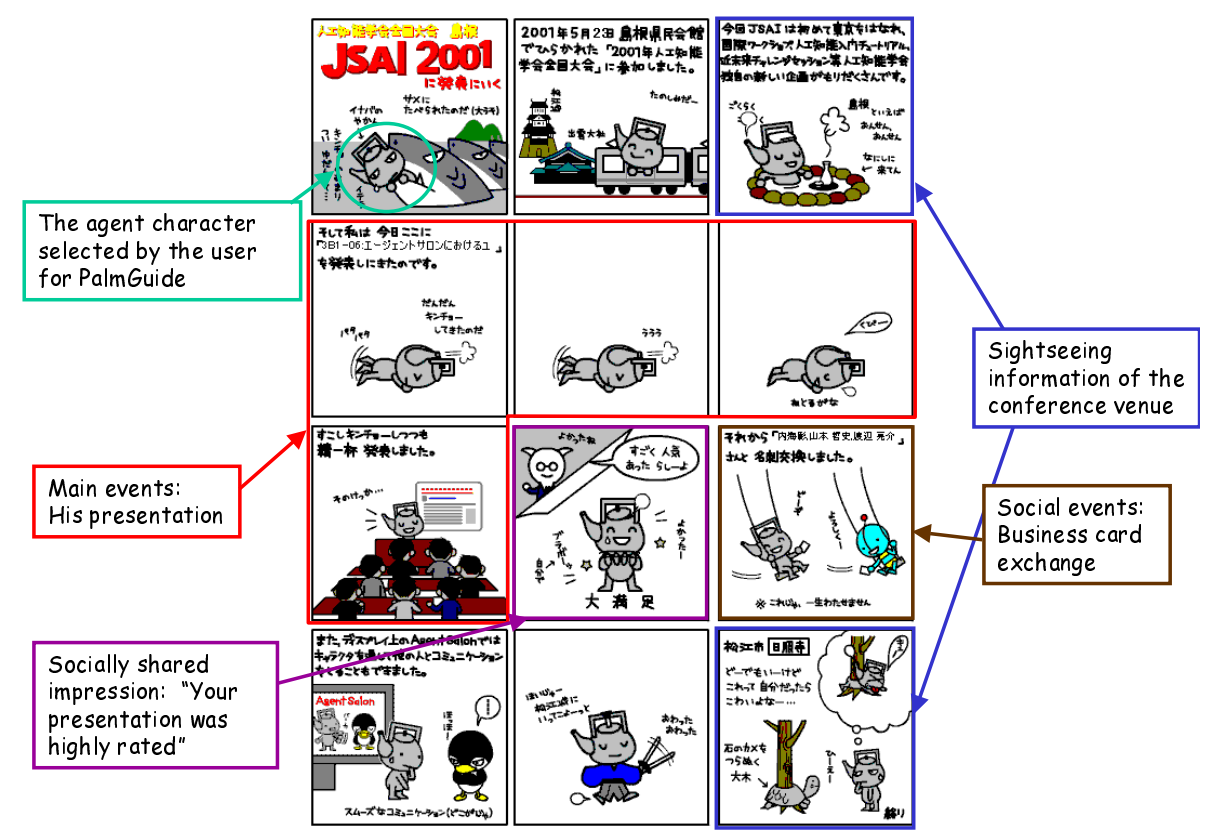

Fig. 8. Example of generated comic diary (for a presenter).

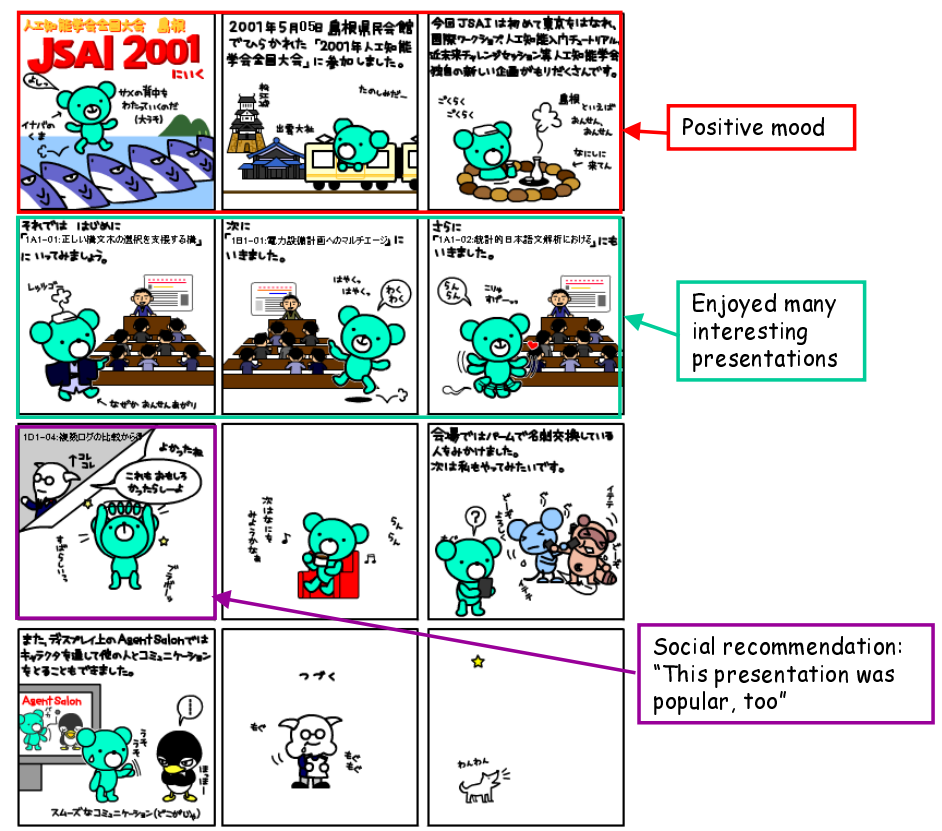

Fig. 9. Example of generated comic diary (active attendance). 


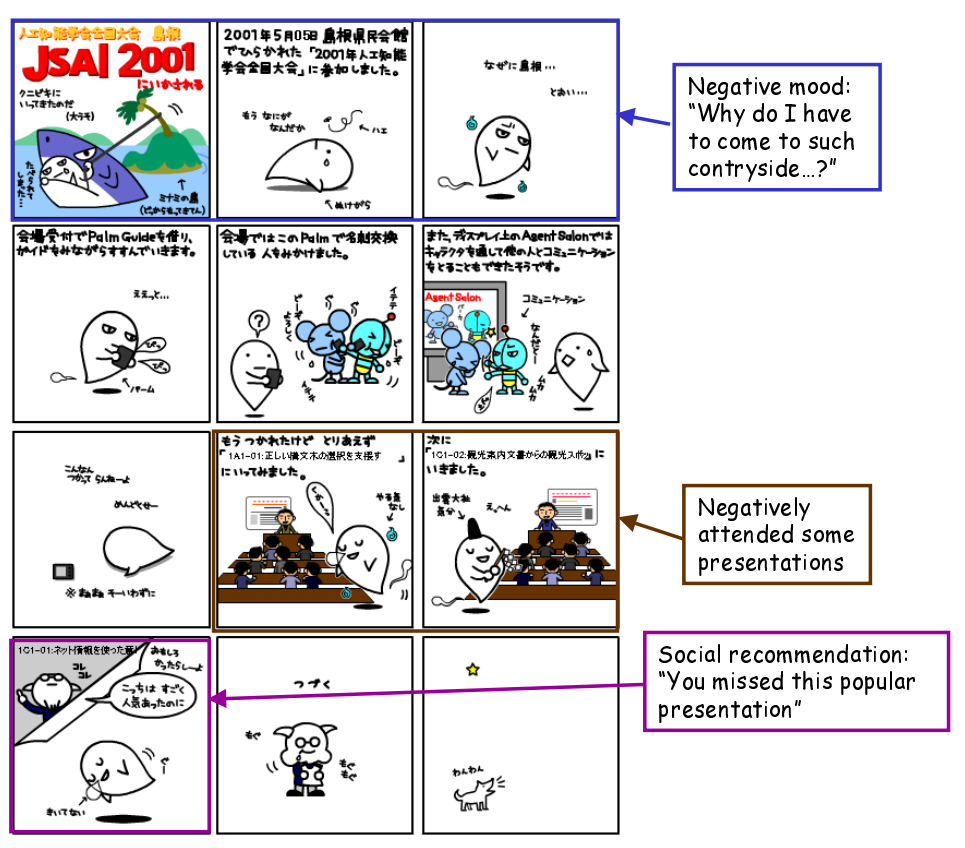

Fig. 10. Example of generated comic diary (non-active attendance).

was not enough for statistical significance, but we were still able to find some interesting tendencies.

The effects of using ComicDiary that we mostly expected were "users want to show their comic diaries to other people" and "it activates conversations among people." To verify these hypotheses, we asked users how many people they had shown their printed comic diaries to. Three users said "showed to nobody," one said "showed to one person," three said "showed to three people," one said "showed to four people," and four said "showed to more than five people." Four users "did not receive the printed version." That is, two thirds of the users who received the printed diaries showed them to more than three people.

To the question "do you think the comic diary encourage(d) conversations with the person(s) to whom you show(ed) the comic?", 14 of the 16 users replied "yes." Here, those who showed their comic diaries to nobody or did not bring them back were asked to answer by supposing that they had showed them to someone.

We also asked a question about the contents of the comics. To the question "does the comic exactly represent your memory?", two users answered "exactly represents," seven answered "fairly represents," five answered "moderate," two answered "not represents well," and nobody answered "not at all." We can thus say that the diaries will augment the users' memories. 
It is interesting that seven users answered "exactly represents," or "fairly represents" while ten users among the 16 answerers were "presenters." This means that we could observe a tendency of "presenters" to evaluate the comic diaries more highly than "attendances (with no presentation)." From the result, we learned that the presentation scene, which is a highly personalized episode, helped to increase the reality of the comics.

Next, we discuss the effect of comic representation in comparison with another representation, i.e., a simple list of personal data accumulated in the PalmGuide devices. We have already provided our digital assistant users with the ability to automatically create touring diaries in hyper-text style (Fig. 11) $[5]$.
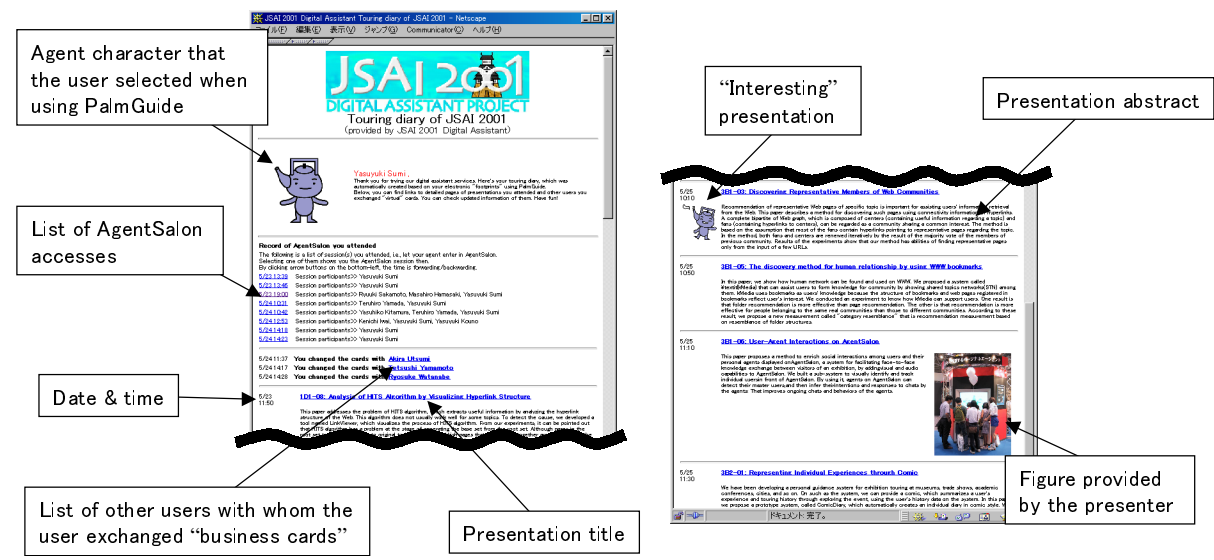

Fig. 11. A diary in hyper-text style.

As we can see in Fig. 11, the hyper-text style diaries accurately show all data of the user's touring history and interactions with other PalmGuide users. The user can get detailed information by following the links of presentations that the user attended and people with whom the user exchanged a virtual business card. The hyper-text diary would seem better able to carefully explore information related to the user's experience. However, we thought this format would not be suitable for light-heartedly sharing one's impressions and experiences with other people.

On the other hand, ComicDiary aims to represent its users' personal impressions by summarizing their experiences from fragmentary data. We assumed that comic diaries would be superior for encouraging conversations among people.

In fact, to the question "which is better to show to other people, comic style or hyper-text style?" ten users replied "comic style," nobody replied "hypertext style," four replied "neither," and two replied "cannot decide." That is, the 
comic style was obviously better accepted for experience sharing than hyper-text style.

\section{Another Prototyping System as Online Service}

We successfully integrated the first prototype of ComicDiary used for JSAI 2001 in May 2001 (JSAI version) with the rest of the digital assistant system. Comic diaries were enjoyed by most PalmGuide users, and the diaries could be electronically accessed via the Web after the conference. PalmGuide users, however, were only 52 of about 500 conference participants. Therefore, it was difficult to expect the effect of users showing their comic diaries to each other after the conference because diaries were provided to only PalmGuide users.

In order to increase system accessibility, we prototyped another version (SIGGRAPH version) as a Web service for an unofficial demonstration at SIGGRAPH 2001, an international conference held in August 2001 in Los Angeles. Because we could not obtain the conference data beforehand and provide participants with PalmGuide devices we abandoned the idea of acquiring personal data for comic diaries by PalmGuide. Instead, we prepared a short questionnaire to acquire each user's personal data for personalizing his/her comic diary.

We prepared about ten easy-to-answer questions such as "Could you find any interesting presentations in this year's SIGGRAPH?", "What is the title of the presentation?", and "Did you attend a party during the conference period?" as shown in Fig. 12 (1). The SIGGRAPH version displayed personalized diaries by modeling a user based on the answers to the questions.

Fig. $12(2)$ is an example of a generated comic diary. Similar to the JSAI version, we tried to represent the conference atmosphere by embedding scenes of the conference venue and popular presentations at the conference ${ }^{2}$.

In the JSAI version, we prepared three types of story outlines, i.e., for presenters, active attendees, and non-active attendees. Each type had a predefined template for scenes, and the number of comic frames was fixed at 12. Accordingly, once the outline was selected from the three types, comic variation was limited to the personalization of individual frames, such as the word layer's personalization, rather than drastic changes in the story outline.

In contrast, the SIGGRAPH version simply defined the maximum number of comic frames (12 frames) and adopted a more flexible strategy. Each scene (of presentation, party, sightseeing, etc.) has a different number of frames, and there are mutual dependencies among the scenes to be selected for a diary as shown in Fig. 5. Based on the question-and-answer dialogue between the system and the user, the user model is gradually built. Then, the next question is selected according to the current user model. Also, scene candidates are collected according to the user model, and then a comic result is displayed when the frame number closes at 12 .

\footnotetext{
${ }^{2}$ Because we did not have statistical user data as in the JSAI version, we updated a list of popular presentations according to our subjective impressions, and the system randomly selected one presentation from the list when generating a comic diary.
} 


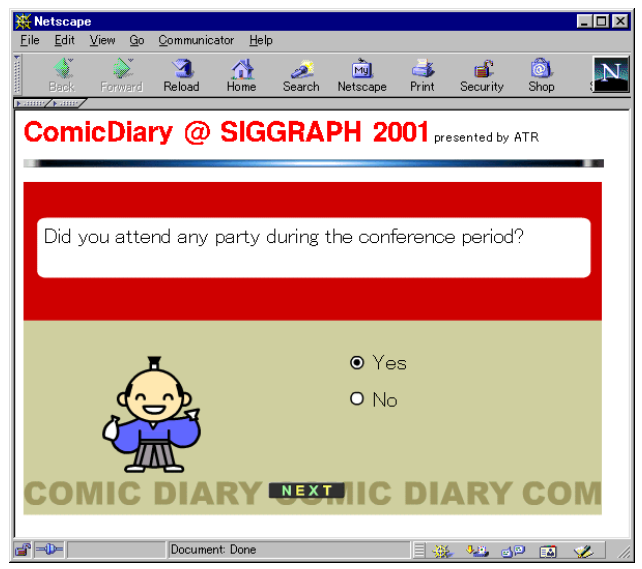

(1) Questionnaire for acquiring the user's personal data.

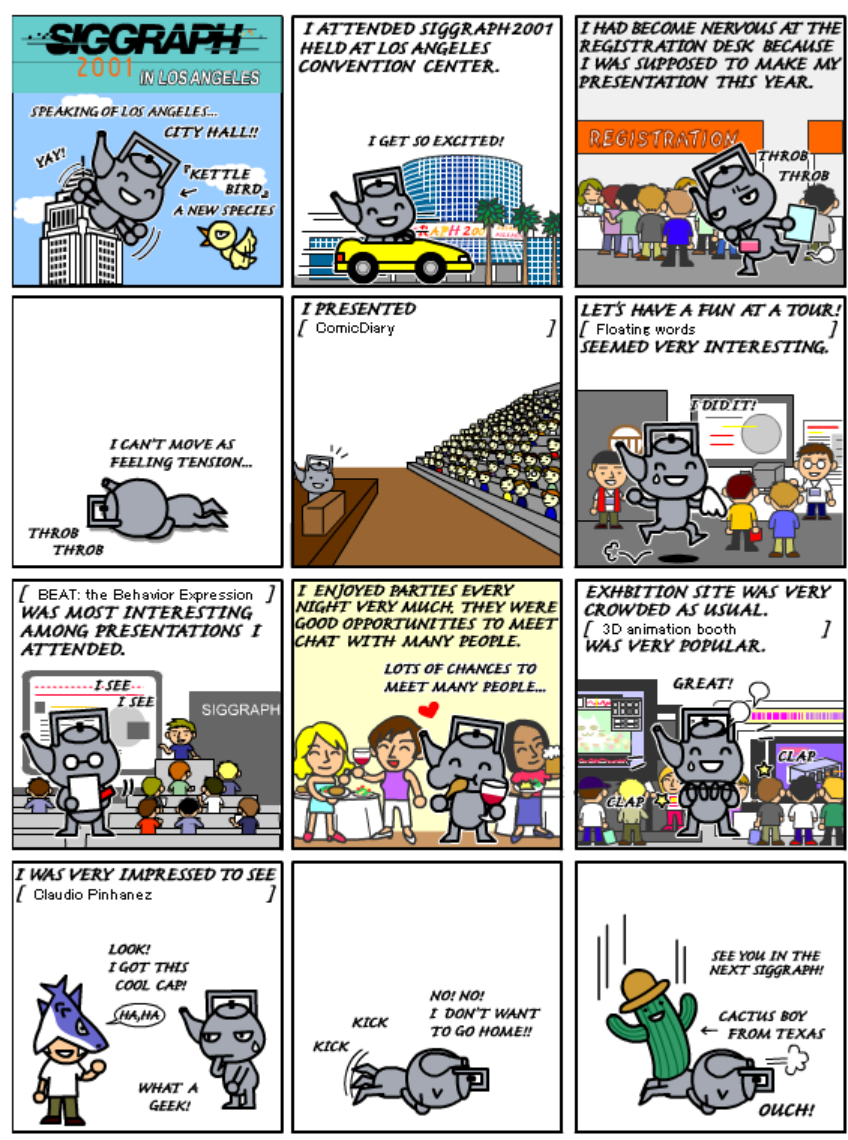

(2) Example of generated comic diary.

Fig. 12. Online version of ComicDiary. 
In the SIGGRAPH version, we added a function featuring network capability. The users could input their e-mail addresses when they liked displayed comics and then receive e-mail messages including URLs of the comics. By using this function, we could observe that some users revisited to their comics several times and other users linked the given URLs to their own homepages to show their (pseudo) experiences at SIGGRAPH 2001. Also, it would be possible for users to inform their friends of their comic diaries by inputting the friends' e-mail addresses, although such usage was not originally intended. In this way, the online version of ComicDiary could increase its capability as a medium to share the users' experiences by showing their comic diaries to each other.

\section{Future Works}

Two issues must be approached in future works: one concerns knowledge processing techniques, and the other concerns techniques of computer graphics.

Regarding knowledge processing techniques, we want to build a framework of flexible user-modeling from the user data and increase the variety of stories generated according to the user-model. Currently, we ad hoc embed dependencies among scenes in the program as shown in Fig. 5, and this decreases the flexibility of the system. An authoring tool to maintain heuristic rules by comic experts themselves would be an appropriate solution because the authoring work of the dependencies among scenes is an expert skill of comics designers.

Regarding techniques of computer graphics, we want to employ a morphable model of characters to decrease the cost of preparing comic parts for a character. Also, we are interested in an approach to exploit resources provided by event participants (e.g., character illustrations and pictures/videos recorded during the event) as raw materials of comics. This would also be interesting in terms of sharing knowledge and experiences among the members of a community.

\section{Conclusions}

We proposed a system called ComicDiary that represents individual experiences and interests in a comics style. We regard ComicDiary as the first prototype of a tool to summarize data ubiquitously obtained and to facilitate knowledge sharing among users based on the shared contexts. The system was prototyped as a digital assistant service for conference participants. We also reported user evaluation of the effect of information sharing among the users. The current evaluation, however, is only a small-scale survey on the effect of facilitating conversations between ComicDiary users and non-users in terms of the usefulness of comic representation. We would like to develop and evaluate a new function to directly support interactions among the users. 


\section{Acknowledgments}

We would like to thank Ryohei Nakatsu and Norihiro Hagita for their helpful discussion and Yasuhiro Tanaka and Tetsushi Yamamoto for their technical support. This research was supported in part by the Telecommunications Advancement Organization of Japan.

\section{References}

1. Yasuyuki Sumi and Kenji Mase. Supporting the awareness of shared interests and experiences in communities. International Journal of Human-Computer Studies, 56(1):127-146, 2002.

2. Mik Lamming and Mike Flynn. "Forget-me-not" Intimate computing in support of human memory. In Proceedings of International Symposium on Next Generation Human Interface '94, pages 150-158. FRIEND21, 1994.

3. Gregory D. Abowd, Christopher G. Atkeson, Ami Feinstein, Cindy Hmelo, Rob Kooper, Sue Long, Nitin Sawhney, and Mikiya Tani. Teaching and learning as multimedia authoring: The classroom 2000 project. In ACM Multimedia 96, pages 187-198. ACM, 1996.

4. Brian Clarkson, Kenji Mase, and Alex Pentland. The familiar: A living diary and companion. In CHI 2001 Extended Abstracts, pages 271-272. ACM, 2001.

5. Yasuyuki Sumi and Kenji Mase. Digital assistant for supporting conference participants: An attempt to combine mobile, ubiquitous and Web computing. In Proceedings of Ubicomp 2001 (Springer LNCS2201), pages 156-175, 2001.

6. Elizabeth D. Mynatt, Jim Rowan, Annie Jacobs, and Sarah Craighill. Digital family portraits: Supporting peace of mind for extended family members. In Proceedings of CHI 2001, pages 333-340. ACM, 2001.

7. D. E. Rumelhart. Notes on a schema for stories. In D. Bobrow and A. Collins, editors, Representation and Understanding: Studies in Cognitive Science. Academic Press, 1975.

8. R. C. Schank and R. P. Abelson. Script, Plans, Goals, and Understanding. Lawrence Erlbaum, 1977.

9. N. Okada and T. Endo. Story generation based on dynamics of the mind. Computational Intelligence, 8(1):123-160, 1992.

10. Takashi Ogata, Koichi Hori, and Setsuo Ohsuga. A besic framework for narrative conceptual structure generation based on narrative techniques and strategies. Journal of Japanese Society for Artificial Intelligence, 11(1):148-159, 1996. in Japanese.

11. David Kurlander, Tim Skelly, and David Salesin. Comic Chat. In Proceedings of SIGGRAPH'96, pages 225-236. ACM, 1996.

12. Shingo Uchihashi, Jonathan Foote, Andreas Girgensohn, and John Boreczky. Video Manga: Generating semantically meaningful video summaries. In Proceedings of Multimedia'99, pages 383-392. ACM, 1999.

13. Yuji Ayatsuka, Nobuyuki Matsushita, and Jun Rekimoto. ChatScape: A visual informal communication tool in communities. In CHI 2001 Extended Abstracts, pages 327-328. ACM, 2001. 